\title{
Study of Fungal Growth in Plaque Psoriasis Patients \\ E.M.Sanad $^{1}$, A.M.Hamed ${ }^{1}$, R.A.El Sayed ${ }^{2}$ and N.N.Nazmy ${ }^{1}$
}

${ }^{1}$ Dermatology \& Andrology Department, Dept., Faculty of Medicine, Benha Univ., Benha, Egypt

${ }^{2}$ Medical Microbiology \& Immunology, Dept., Faculty of Medicine, Benha Univ., Benha, Egypt

Email:nader.zaki@fmed.bu.edu.eg

\begin{abstract}
Background: Psoriasis is a common, chronic, immune-mediated disease manifested mainly as skin lesions and extracutaneous comorbidities associated with systemic inflammation. Typical skin manifestations of psoriasis include erythematous, indurated, and scaling plaques that are painful, itchy, and have a burning sensation. Fungi as Malassezia and Candida albicans have been implicated in the pathogenesis of psoriasis. Aim of work: is to study the association between the presence of different fungal growth and psoriasis. Patients and Methods: This study was carried out on a total of 30 patients clinically diagnosed to have psoriasis, attending the outpatient clinic of the Dermatology and Andrology Department of Benha University Hospital, from April 2019 to July 2020. All patients were subjected to complete history taking, general examination, and dermatologic examination to confirm the diagnosis. An informed consent approved by the Research Ethics Committee at Faculty of Medicine Benha University was taken from all individuals before being enrolled in this study. From the lesions, scales were collected by gentle scraping, examined microscopically for detection of any fungal growth using $10 \%$ potassium hydroxide $(\mathrm{KOH})$ and then cultured on Sabouraud's Dextrose Agar (SDA) with and without cyclohexamide and Dixon's medium for identification of any fungal growth. Results: The mean age of patients was 38.3, twenty patients were males and 15 patients reported to be smokers. Only seven patients had positive family history. Most patients had gradual onset and course of exacerbation and remission. Twenty two cases of psoriasis showed positive $\mathrm{KOH}$ and culture for fungal growth. 10 cases were positive for Aspergillus, 7 for Candida, 4 for Malassezia, and only one case for Dermatophytes. Conclusion: Fungal infection may play a role in the pathogenesis of psoriasis, its prevalence is still a matter of debate and its exact role is in need for more research to be revealed.
\end{abstract}

Keywords: Psoriasis, fungal growth, Aspergillus, Candida, Malassezia.

\section{Introduction}

Psoriasis is a common, chronic, immune-mediated disease characterized by epidermal hyperproliferation, an intraepidermal accumulation of neutrophils, and dermal inflammatory cell infiltrates that are composed of dendritic cells and different types of T cells [2].

Psoriasis is manifested mainly as skin lesions and extracutaneous comorbidities associated with systemic inflammation [3]. Typical skin manifestations of psoriasis include erythematous, indurated, and scaling plaques that are painful, itchy, and have a burning sensation. Psoriasis decreases patients' quality of life due to unpleasant symptoms and related public stigma. The unpleasant skin appearance contributes to reduced employment levels and thus, affects the financial status of patients [4].

Fungi have been implicated in stimulating skinassociated lymphoid tissue and certain fungi such as Malassezia and C. albicans have been found to be associated with psoriasis [8].

\section{Aim of work}

Aim of work:Is to study the association between the presence of different fungal growth and psoriasis.

\section{Patients and Methods}

Table (1) Demographic data and smoking of studied patients.
The present study was carried out on a total of 30 individuals attending the outpatient clinic of the Dermatology and Andrology Department of Benha University Hospital, from April 2019 to July 2020.

Patients included in this study were diagnosed clinically to have psoriasis, while any patient with other dermatologic or systemic disease was excluded from the study.

All patients were subjected to complete history taking, general examination, and dermatologic examination to confirm the diagnosis. An informed consent approved by the Research Ethics Committee at Faculty of Medicine Benha University was taken from all individuals before being enrolled in this study.

Scales were collected from the lesions by gentle scraping using sterile scalpel blade and then examined microscopically for fungal growth by using $10 \%$ potassium hydroxide $(\mathrm{KOH})$ and also specimens were cultured in Sabouraud's Dextrose Agar (SDA) for identification of any fungal growth.

\section{Results}

The mean age of patients was 38.3 , twenty patients were males and 15 patients reported to be smokers Table(1)

\begin{tabular}{llccc}
\hline & & \multicolumn{2}{c}{ Psoriasis } & P \\
Age (years) & Mean \pm SD & $\mathbf{3 8 . 3}$ & $\pm \mathbf{3 0}$ & $\mathbf{0 . 8 4}$ \\
Males & $\mathbf{N}, \%$ & $\mathbf{2 0}$ & $60 \%$ & $\mathbf{1}$ \\
Females & $\mathbf{N}, \%$ & $\mathbf{1 0}$ & $40 \%$ & \multicolumn{1}{c}{} \\
\hline
\end{tabular}




\begin{tabular}{lcccc}
\hline Positive family history & $\mathbf{N}, \boldsymbol{\%}$ & $\mathbf{5}$ & $16.6 \%$ & 0.025 \\
Smoking & $\mathbf{N}, \boldsymbol{\%}$ & 15 & $50 \%$ & $<0.001$ \\
\hline
\end{tabular}

Only seven patients reported positive family history, most patients had gradual onset and course of exacerbation and remission Table (2).

Table (2) Clinical features of all psoriasis cases.

\begin{tabular}{|c|c|c|c|c|}
\hline & & & \multicolumn{2}{|c|}{$\begin{array}{c}\text { Psoriasis } \\
\mathbf{N}=\mathbf{3 0}\end{array}$} \\
\hline \multicolumn{2}{|l|}{ Positive family history } & $\mathrm{N}, \%$ & 7 & $23.3 \%$ \\
\hline \multirow[t]{2}{*}{ Onset } & Gradual & $\mathbf{N}, \%$ & 28 & $93.3 \%$ \\
\hline & Acute & $\mathbf{N}, \%$ & 2 & $6.4 \%$ \\
\hline \multirow[t]{2}{*}{ Course } & Remission and exacerbation & $\mathbf{N}, \%$ & 25 & $83.3 \%$ \\
\hline & Progressive & $\mathbf{N}, \%$ & 5 & $16.7 \%$ \\
\hline \multirow[t]{2}{*}{ Distribution } & Generalized & $\mathbf{N}, \%$ & 14 & $46.6 \%$ \\
\hline & Localized & $\mathbf{N}, \%$ & 16 & $50.4 \%$ \\
\hline
\end{tabular}

Twenty two cases of psoriasis showed positive $\mathrm{KOH}$ and culture for fungual growth. Ten cases were positive for Aspergillus, 7 for candida, 4 for Malassezia, and only one case for dermatophyte Table (3).

Table (3) Fungal growth in studied patients

\begin{tabular}{|c|c|c|c|c|}
\hline & & & \multicolumn{2}{|c|}{$\begin{array}{c}\text { Psoriasis } \\
\mathrm{N}=\mathbf{3 0}\end{array}$} \\
\hline & & & $\mathrm{N}$ & $\%$ \\
\hline \multicolumn{2}{|c|}{ Direct Examination (KOH 10\%) } & $\mathrm{N}, \%$ & 22 & $73.3 \%$ \\
\hline \multirow[t]{4}{*}{ Identified fungal Genus } & Aspergillus & $\mathbf{N}, \%$ & 10 & $45 \%$ \\
\hline & Candida & $\mathrm{N}, \%$ & 7 & $31 \%$ \\
\hline & Malassezia & $\mathbf{N}, \%$ & 4 & $18 \%$ \\
\hline & Dermatophytes & $\mathbf{N}, \%$ & 1 & $4.5 \%$ \\
\hline
\end{tabular}

\section{Discussion}

Many studies have reported an association between psoriasis and different infections. It was found that Staphylococcus aureus is associated with the development of psoriasis [1]. Moreover, the severity of psoriasis significantly correlates with enterotoxin production by the isolated Staphylococcus aureus strains [9].

The role of different microorganisms in the pathogenesis of psoriasis is still unknown. However, it was proposed that that role is through stimulating an immune response that in turn causes a state of continuous inflammation.

The results of the present study revealed that 22 cases of psoriasis showed positive $\mathrm{KOH}$ and culture for fungual growth confirming the possible role of fungal infection in the pathogenesis of psoriasis.

Our results also revealed $45 \%$ positive cases with Aspergillus. Traditionally, laboratory diagnosis of Aspergillus species relied on morphology-based identification methods to differentiate the types of Aspergillus species. Diagnostic criteria include the recognition of asexual or sexual structures and their characteristics such as shape, size, color, ornamentation and/or mode of attachment [6].

Thirty one \% of psoriasis cases showed positive $\mathrm{KOH}$ results for candida. Most pathogenic Candida species are dimorphic fungi, existing as yeast or pseudohyphal and hyphal forms. Dimorphism is a key feature of virulence of
C. albicans, and the tissue-invasive hyphal form is generally the most pathogenic one [5]. The recognition of different morphotypes of C. albicans permits discrimination between commensal and pathogenic disease-causing forms [7].

\section{Conclusion}

Fungal infection may play a role in the pathogenesis of psoriasis, its prevalence is still a matter of debate, and also the exact role is in need for more research to be revealed.

\section{References}

[1] A.V.Alekseyenko, G.I.Perez-Perez, A.De Souza, B.Strober, Z.Gao. Community differentiation of the cutaneous microbiota in psoriasis. Microbiome.vol.1,pp.31,2013.

[2] W.H.Boehncke, and M.P.Schön. Psoriasis. Lancet.vol.386,pp.983-994,2015.

[3] J.E.Greb, A.M.Goldminz, J.T.Elder, M.G.Lebwohl, D.D.Gladman. Psoriasis. Nat Rev Dis Primers.vol. 2,pp.16082,2016.

[4] T.Hawro, A.Zalewska, M.Hawro, A.Kaszuba, M.Krolikowska. Impact of psoriasis severity on family income and quality of life. J Eur Acad Dermatol. Venereol..vol.29,pp.438-443,2015.

[5] S.W.Kashem, B.Z.Igyarto, M.Gerami-Nejad, Y.Kumamoto, J.Mohammed. Candida albicans 
morphology and dendritic cell subsets determine $\mathrm{T}$ helper cell differentiation. Immunity.vol. 42,pp.356366, 2015.

[6] C.Kosmidis, P.Newton, E.G. Muldoon and D.W.Denning. Chronic fibrosing pulmonary aspergillosis: a cause of 'destroyed lung' syndrome. Infect Dis.vol.49(4), pp.296-301,2017.

[7] L.Monin, and S.L.Gaffen. IL-17 family cytokines: signaling mechanisms, biological activities and therapeutic implications. Cold Spring Harb Perspect Biol.vol. 10(4),pp.a028522, 2018.

[8] A.Takemoto, O.Cho, Y.Morohoshi, T.Sugita, and M.Muto. Molecular characterization of the skin fungal microbiome in patients with psoriasis. J. Dermatol.vol. 42,pp.166-170,2015.

[9] J.E.E.Totté, W.T.van der Feltz, M.Hennekam, A.van Belkum, E.J.van Zuuren. Prevalence and odds of Staphylococcus aureus carriage in atopic dermatitis: a systematic review and meta-analysis. $\mathrm{Br}$. J Dermatol.vol.175(4),pp.687-695,2016. 